\title{
New Intelligent Control of Auto-self Defensive Unmanned Aerial Vehicle
}

\author{
${ }^{1}$ Marwa A. El-Diwiny, ${ }^{2}$ Abou-Hashema M. El-sayed \\ ${ }^{1}$ Mechatronics and Industrial Robotics, Minya University, 61519, Egypt \\ ${ }^{2}$ Faculty of Engineering,Mechatronics, Minya university, 61519, Egypt \\ marwa.eldiwiny@eng.miniauniv.edu.eg, abouhashema@mu.edu.eg
}

\begin{abstract}
This paper handle a new terminology of the field of the unmanned aerial vehicle is Auto-self defensive UAV, by using new configuration of $x$-band Doppler surface distribution. The aim of this research is to make UAV can avoid any directed missiles at small time delay before the collision. The intelligent control will process the data of the hypothetical missiles by using supercomputing and send it to the inertial navigation system to correct the path of UAV every sample time against the missile. The goal is to make intelligent $U A V$ that can maneuver the missile and never collide with it.
\end{abstract}

Key-Words Unmanned aerial vehicle, Inertial navigation system, Ku-band frequency RADAR, Arduino Mega 2650, ArduIMU V-3.

\section{INTRODUCTION}

Over the years airborne radars have proven their value as wide-area, nearly all-weather surveillance tools. Typically, airborne radars are large systems mounted in manned aircraft. Lincoln Laboratory, however, has built a very capable radar system that is compact and lightweight; the radar has been integrated into an unmanned air vehicle(UAV). The work is sponsored by the Army's Harry Diamond Laboratories and the Defense Advanced Research Projects Agency (DARPA). The onboard processing permits the use of a UAV data link that transmits kilobits per second of moving-target reports instead of tens of megabits per second of raw radar data. The system-the airborne portion of which weighs only $110 \mathrm{lb}$-detects and tracks moving vehicles such as tanks, trucks, and low-flying helicopters out to a range of $15 \mathrm{~km}$, an classifies them a shorter ranges.

Unmanned air vehicles (UAV) have proven to be useful for observing activity on the ground without placing an air crew at risk. Using television cameras aboard small $U A V s$, the Israelis have successfully penetrated hostile air defenses and observed ground activity in enemy Territories [1].

\section{NEW AUTO SELF-DEFENSIVE UAV}

The new UAV is fully capable avoiding directed air to air missile or surface to air missile by using new RADAR configuration for achieving it at small time delay. In this research the hypothesis that there are multiple missiles at directed to $U A V$, by using new configuration the $U A V$ will be out of the collision with the directed missile by using high compute computation. The aim of this hypothesis making intelligent UAV that never collides with directed missiles enemy.

\section{THE MODEL USED FOR HYPOTHESIS}

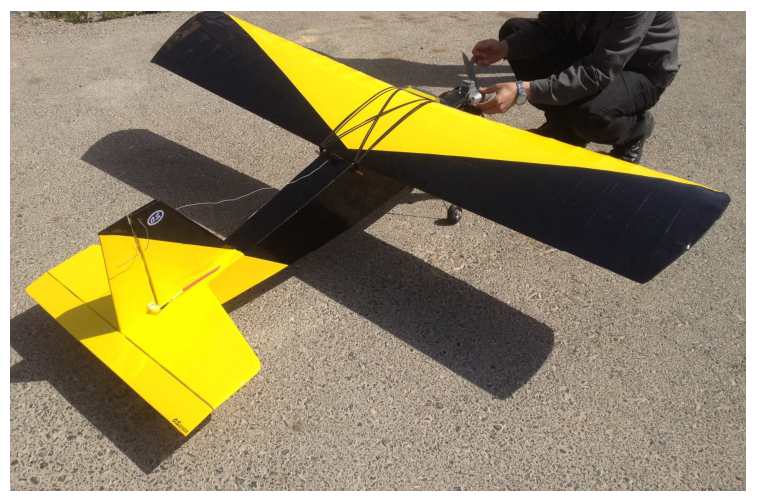

Fig.1.RC KADET LT_25

The model used is RC KADET LT_25 fixed wing, The model parameters as shown at Table.1 [2].By using wind tunnel Autodesk Falcon for the model parameters as shown at Fig. 2

TABLE I

PLATFORM PARAMETER

$\begin{array}{lll}\text { Wingspan } & 63 \mathrm{in} & 1600 \mathrm{~mm} \\ \text { Wing Area } & 724 \mathrm{in}^{2} & 46.71 \mathrm{dm}^{2} \\ \text { Weight } & 4-41 / 4 \mathrm{lb} & 1814-1928 \mathrm{~g} \\ \text { Wing Loading } & 12-13 \mathrm{oz} / \mathrm{ft}^{2} & 3662-3967 \mathrm{~g} / \mathrm{m}^{2}\end{array}$


Radio Required 4-Channel

$.25-.32 \mathrm{in}^{3}\left(4.1-5.2 \mathrm{~cm}^{3}\right) \quad 2-$ Stroke $20-.26 \mathrm{in}^{3}\left(3.3-4.3 \mathrm{~cm}^{3}\right)$-stroke

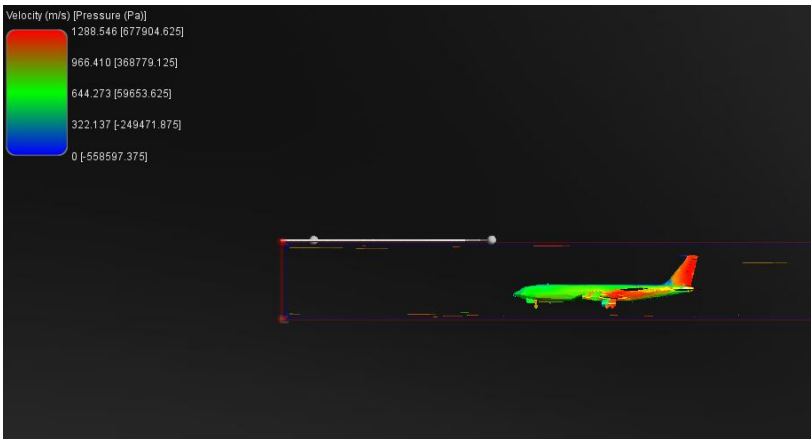

Fig.2.Wind Tunnel-Autodesk Falcon

\section{INERTIAL NAVIGATION SYSTEMS}

Inertial navigation is a self-contained navigation technique in which measurements provided by accelerometers and gyroscopes are used to track the position and orientation of an object relative to a known starting point, orientation and velocity. Inertial measurement units (IMUs) typically contain three orthogonal rate-gyroscopes and three orthogonal accelerometers, measuring angular velocity and linear acceleration respectively. By processing signals from these devices it is possible to track the position and orientation of a device, Inertial navigation is used in a wide range of applications including the navigation of aircraft, tactical and strategic missiles, spacecraft, submarines and ships. Recent advances in the construction of MEMS devices have made it possible to manufacture small and light inertial navigation systems. These advances have widened the range of possible applications to include areas such as human and animal motion capture. uses, and the two main varieties of inertialnavigationsystem [3].

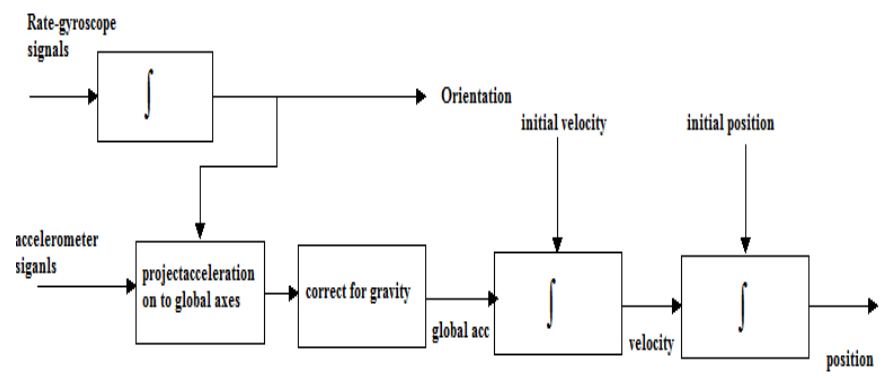

Fig.3.Inertial navigation system

\section{A. Strap down system}

In strap down systems the inertial sensors are mounted rigidly onto the device, and therefore output quantities measured in the body frame rather than the global frame. To keep track of orientation the signals from the rate gyroscopes are 'integrated'. To track position the three accelerometer signals are resolved into global coordinates using the known orientation, as determined by the integration of the gyro signals. The global acceleration signals are then integrated as in the stable [4].

\section{A.1.1. Inertial Navigation System Procedure}

ArduIMU is not an Autopilot. It's just an orientation solution (the heart of an Autopilot) and can be used on anything where you want to know its orientation in respect to the ground.

ArduIMU V3 features the new MPU-6000 that includes 3 axis gyros \& accells built-in, the latest 3 axis $I 2 C$ magnetometer HMC-5883L and the classic but very robust Arduino Atmega328 running at 16Mhz[5].

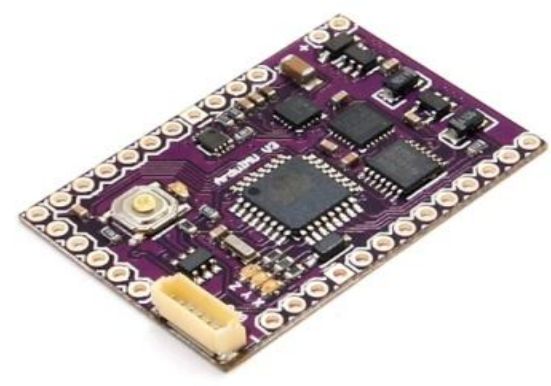

Fig.4.ArduIMU -V3

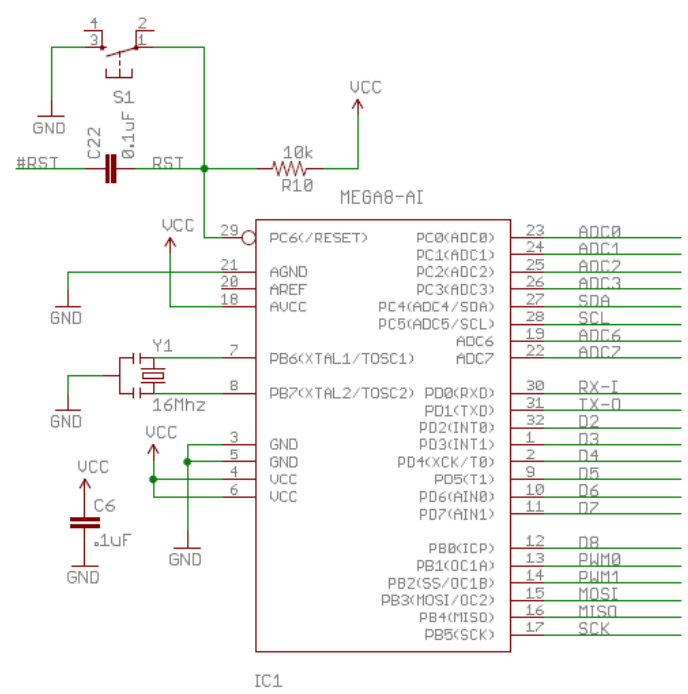

Fig. 6.ArduIM V-3 Circuit 


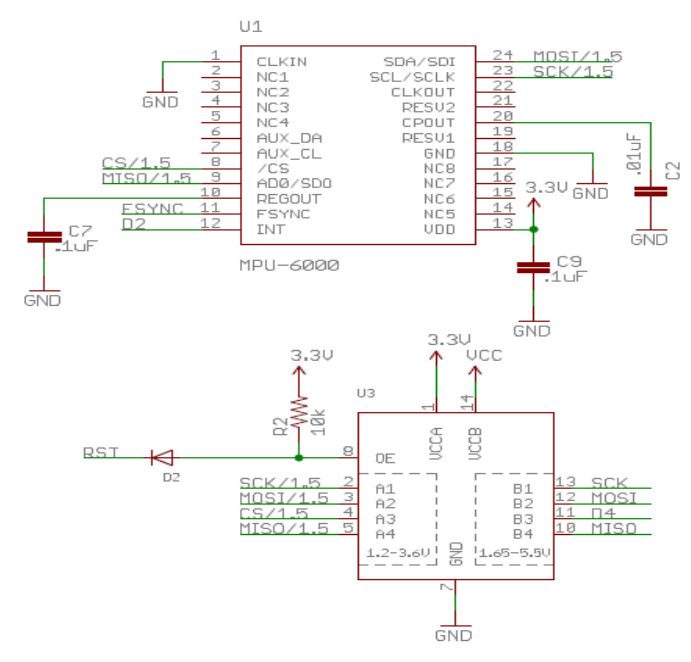

Fig.7.MPU-6000 Circuit

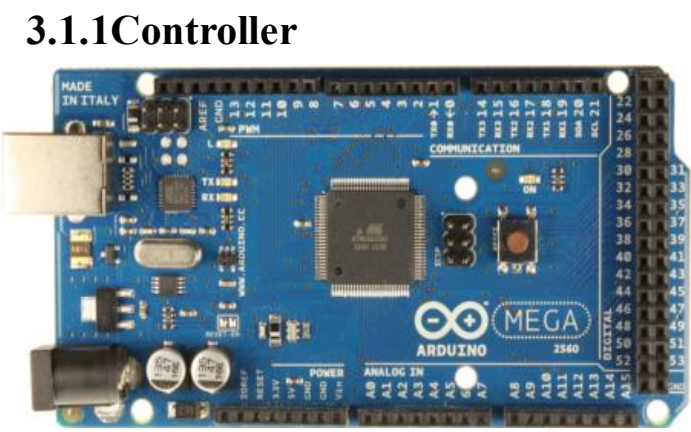

Fig.8.Arduino Mega 2650

\section{TEST RESULTS}

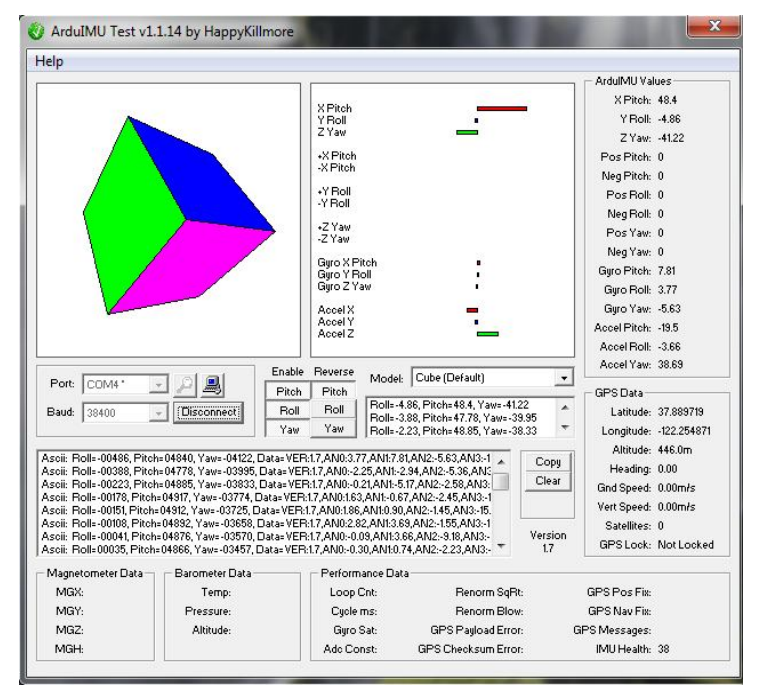

Fig. 10.ArduIMU Test

\section{NEW RADAR UAV CONFIGURATION}

In this paper, a new UAV RADAR configuration hypothesized to make the delay time very small it is proposed that four ballistic missiles targeted to the $U A V$. it is needed to determine the speed and the coordinates of the ballistic missile at low time delay (warning system). The technique used K-band Doppler but in this research the radar antenna concentrative 90 degree. The platform used $R C$ KADET LT-25 will be divided in four parts. In each part K-band Doppler will detect its region.The schematic diagram of the four radar system $s$ will register each reading after processing the comparators will be used to compare each radar's reading. By using supercomputing the controller has two choices to remain the first reading or change the second reading if it will be in not safe coordinates to UAV. The aim to make UAV more intelligent and early warning system that will give the opposite coordinates to UAV immediately. Consequently the UAV will never be hit by any missile.



Fig.11. New RADARUAV architecture configuration 


\section{CONCLUSION}

Unmanned Aerial Vehicles are an exciting field in the world of aviation, with new discoveries and proposed uses being documented daily. Over the next 16 years, UAVs will become a significant component of military, civil, and perhaps even commercial aviation. However, the very dynamic nature of the field also creates a significant amount of uncertainty. The wide range of UAV physical and performance characteristics, many of which will be very unlike any current aircraft, will place additional challenges on an air traffic management system already under great strain. However, many of the new paradigms being considered for the future NAS will likely facilitate the routine and safe entry of UAV operations into civil airspace. The information management system, through shared situational awareness, will allow all users of the NAS to know the location and intent of other aircraft (both manned and unmanned.

\section{REFERENCES}

[1] C.E. Schwartz, T.G. Bryant. J.H. Cosgrove, G.B. Morse, andJ.K. NoonanH. Wu, D. Sun, and Z. Zhou, "Micro air vehicle: Configuration, analysis, fabrication, and test," IEEE/ASME Trans. on Mechatronics, vol. 9, no. 1, pp. 108117, 2004.

[2] http://www.sigmfg.com

[3] H. Chao, M. Baumann, A. Jensen, Y. Q. Chen, y .Cao, W. Ren, and M. McKee, "Bandreconfigurable multi-UAVbased cooperative remote sensing for real-time water management and distributed irrigation control," Proc. IFAC World Congress, pp. 11744-11749, 2008.

[4] J. Pappalardo, "Unmanned aircraft roadmap reflects changing priorities," National Defense, vol. 87, no. 392, pp. 30, 2003. G. K. Egan, "The use of infrared sensors for absolute attitude determination of unmanned aerial vehicles," Tech. Rep. MECSE-22-2006, Monash University, Australia, 2006.

[5] www.Diydrone.com

[6] www.arduino.cc

\section{Creative Commons Attribution License 4.0 (Attribution 4.0 International, CC BY 4.0)}

This article is published under the terms of the Creative Commons Attribution License 4.0

https://creativecommons.org/licenses/by/4.0/deed.en US 